\title{
Transport properties of interpenetrating phase composites tin-carbon
}

\author{
S. Krcho* \\ KOMPOZITUM Ltd., R@D Department, Dopravná 3A, 95501 Topolčany, Slovak Republic
}

Received 3 September 2015, received in revised form 6 November 2015, accepted 2 December 2015

\begin{abstract}
Interpenetrating phase composites carbon-tin prepared by the pressure infiltration technology were tested in terms of electrical conductivity, thermal conductivity and sound velocity relating to the tin content (up to 25 vol.\%. Sn). Results obtained show that at tin concentration $x_{\mathrm{c}}=7.9 \mathrm{vol} . \%$ the continuous cluster of the tin component in the carbon skeleton is formed. As a result, the percolation of electrons in composites occurred changing the concentration dependence of electrical conductivity from linear to power. Thermal conductivity and sound velocity of composites increased linearly with increase in tin content without being influenced by the tin cluster structure.
\end{abstract}

Key words: interpenetrating phase composites, infiltrated carbon, tin clusters, electron percolation, thermal conductivity, sound velocity

\section{Introduction}

Today, the development of new composite materials and the advancement of science and technology are increasingly focused on the production of complex technical applications. By combining several types of materials, new materials are produced with a wide range of physical parameters that are unachievable at the individual component levels. One such type of material is an interpenetrating phase composite, which consists of tin and carbon components that are interconnected into one composite unit. The material combines metal properties such as good electrical conductivity, good thermal conductivity, ductility, toughness, and chemical stability, with the material properties of carbon such as lubricity, a high modulus of elasticity, chemical stability, dimensional stability, and low thermal expansion.

Some promising applications using this material include sliding seals and bearings for pumps used in the food industry, sliding applications for the transfer of electricity, as well as electrodes used in electrolytic processes. The present study focuses on the transport properties of the tin-carbon composite produced by pressure infiltration of the carbon matrix with liquid tin.
The carbon matrix contains a continuous system of pores [1-3]. The labyrinth of pores is gradually filled with liquid metal resulting in an embedded interpenetrating phase composite wherein the metal forms interconnected clusters [4-5]. The wettability of carbon by liquid tin is low and the samples can not be infiltrated by spontaneous infiltration since the contact angle of liquid tin on a carbon substrate is $156^{\circ}$ [6]. To overcome repulsive capillary forces and intrude liquid metal into carbon pores external gas pressure can be applied.

The electrical conductivity of polycrystalline carbon is usually in the range between $1 \times 10^{4}$ and $20 \times 10^{4} \Omega^{-1} \mathrm{~m}^{-1}[7-9]$. At $22^{\circ} \mathrm{C}$ the electrical conductivity of tin is $794 \times 10^{4} \Omega^{-1} \mathrm{~m}^{-1}$ [10], which is up to 40 to 800 -times greater than the electrical conductivity of the carbon.

Thermal conductivity of carbon consists of the contribution of electrons and phonons to total thermal conductivity:

$$
K=K_{\mathrm{e}}+K_{\mathrm{f}}
$$

where $K$ is the total thermal conductivity, $K_{\mathrm{e}}$ is the contribution of electrons and $K_{\mathrm{f}}$ is the contribution of phonons. The thermal conductivity is propor-

*Corresponding author: tel.: +421 905788 251; fax: +421 3853204 59; e-mail address: krcho@kompozitum.sk 
tional to the mean free path of the crossing phonons and electrons within carbon material [11]. Using the Wiedemann-Franz rule the ratio $K_{\mathrm{e}} / K_{\mathrm{f}}=0.005$ was calculated for polycrystalline carbon to characterize the contribution of phonons and electrons in thermal conductivity [12]. The value obtained shows that the phonon component is dominant in thermal conductivity of polycrystalline carbon.

To determine the sound velocity of carbon-tin composites, the longitudinal elastic oscillations are examined. For pure tin, the velocity of longitudinal sound oscillations is $3320 \mathrm{~m} \mathrm{~s}^{-1}$ [13].

Generally, the transport properties of materials consisting of two different components are a combination of individual material properties. The final properties may be either linearly dependent on the volume fraction of individual components or can exhibit a non-linear pattern. The non-linear dependence of electrical conductivity on the fraction of graphite grains in the composite was reported in $[14,15]$. In both studies, the authors observed the electron percolation effect due to the formation of the continuous cluster from graphite grains. In another study, the coppercarbon samples were prepared from powder raw materials wherein the authors observed the non-linear dependence of electrical conductivity on the volume fraction of copper and the electron percolation effect [16].

The non-linear dependence of transport properties on the proportions of individual components is explained by the percolation theory, first introduced by Broadbent and Hamersley [17], using the theory of bonds known as the "bond problem".

The specific electrical conductivity $\sigma_{\mathrm{c}}$ can be expressed mathematically as:

$$
\sigma_{\mathrm{c}} \sim\left(x-x_{\mathrm{c}}\right)^{t}, \text { for } x \geq x_{\mathrm{c}},
$$

moreover, analogically:

$$
\sigma_{\mathrm{c}}=0, \text { for } x \leq x_{\mathrm{c}} \text {, }
$$

where $t$ is the critical index, $x$ is the concentration of wet (conductive) clusters, and $x_{\mathrm{c}}$ is the critical concentration of wet clusters. The concentration interval of $x>x_{\mathrm{c}}$ is referred to the overcritical region in which the infinite cluster gradually grows.

During the infiltration process, the conductive metal clusters are created in porous carbon skeleton. It is likely that large and interconnected clusters influence electrical and heat conductivity as well as the sound velocity in resultant composites. The goal of the present study is to characterize the above-mentioned transport phenomena in carbon-tin interpenetrated composites prepared by the pressure infiltration technology in relation to the volume fraction and the connectivity (topology) of the tin component.

\section{Experimental procedure}

To study the transport properties of tin-infiltrated carbon material, there were prepared the samples with various content of tin in the carbon matrix. As a porous carbon body, there was used polycrystalline carbon material KU111L commercially produced by KOMPOZITUM Ltd. The typical shape of pores of carbon samples is so called "ink-bottle" type in which the pore entrance is smaller than the average pore diameter. The majority of pore sizes are declared to be $15 \mu \mathrm{m}$. The total pore fraction in KU111L material is $30.2 \%$. Carbon samples were prismatic with dimensions of $20 \times 20 \times 40 \mathrm{~mm}^{3}$. The infiltration of specimens was performed by the gas pressure infiltration technique. A laboratory autoclave KU5W operating at pressures up to $7 \mathrm{MPa}$ and temperatures up to $1400^{\circ} \mathrm{C}$ located at KOMPOZITUM Ltd. was used for infiltration of liquid tin (purity of 99.95\%) into porous KU111L specimens. Each carbon sample was put into the autoclave and evacuated to $50 \mathrm{~Pa}$ to remove the gas from pores. After being degassed, the samples were immersed into liquid metal, and the external gas pressure (argon) was applied. Each sample group was infiltrated at different pressures (0.16.5 $\mathrm{MPa}$ ) to prepare the samples with different tin content. After 10 minutes of the pressure loading the sample was cooled down to the room temperature keeping still the gas pressure and removed from the autoclave. Infiltrated samples were machined to a rod with a diameter of $10 \mathrm{~mm}$ and length of $30 \mathrm{~mm}$. The structure of infiltrated samples was investigated using the optical microscope. The fraction of tin was determined by weighing the samples before and after infiltration, using Sartorius Model MA45 scales. Pore distribution in carbon material was determined by a mercury porosimeter MICROMERITICS Auto Pore. The study of transport phenomena was conducted gradually, by measuring electrical conductivity, heat conductivity, and sound velocity according to varying proportions of carbon and metal. The measurements were done at $23^{\circ} \mathrm{C}$ and $54 \%$ relative air humidity.

Specific electrical conductivity was measured using the four-point method at $1 \mathrm{~A} \mathrm{~cm}^{-2}$ current density. Voltage and current values were recorded by using of an Agilent 34401A nanovoltmeter, with $100 \mathrm{nV}$ sensitivity. The thermal conductivity was measured by using the stationary Stuckes Chasmar method [9], in which the thermostats of the MA TopTech series (JULABO) with a sensitivity of $0.01^{\circ} \mathrm{C}$ were used. Sound velocity was measured with an Echometer 1076 (KARL DEUTSCH) that features a built-in, steel calibration block with a sound velocity of 5920 $\mathrm{m} \mathrm{s}^{-1}$. The frequency of longitudinal oscillations was $2 \mathrm{MHz}$. 


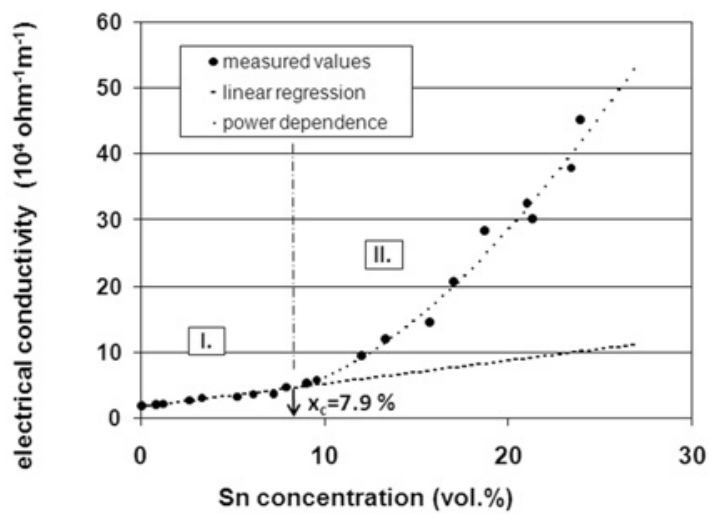

Fig. 1. Dependence of electrical conductivity on Sn volume. Critical Sn concentration $x_{\mathrm{c}}=7.9$ vol. $\% \mathrm{Sn}$.

\section{Results and discussion}

The dependence of the specific electrical conductivity on the tin content is presented in Fig. 1. It is seen that with an increase in the metal concentration the specific electrical conductivity shows a rising tendency. For low metal concentrations, the increase in the electrical conductivity exhibits the linear trend while for higher concentrations the dependence is changed to the power-type curve typical for the percolation of electrons.

The percolation condition, i. e. the contrast between electrical conductivities of individual components, is not met as the background influences the conductivity. The critical cluster and the background represent two conductivity resistances, arranged in parallel, following the Mathiesen's rule. Accordingly, resultant electrical conductivity $\sigma_{\text {ef }}$ is the sum of the background conductivity $\sigma_{\operatorname{lin}}$ and the critical behaviour electrical conductivity $\sigma_{\mathrm{c}}$ :

$$
\sigma_{\mathrm{ef}}=\sigma_{\mathrm{lin}}+\sigma_{\mathrm{c}}
$$

In the linear region we can apply the form:

$$
\sigma_{\operatorname{lin}}=a_{0}+a_{1} x .
$$

By using the standard linear and power regression procedures both the critical tin concentration $x_{\mathrm{c}}=$ 7.9 vol.\% and the critical percolation index $t=1.65$ were determined. At the concentration of 7.9 vol.\% Sn the onset of percolation of electrons in the overcritical region occurs. The experimental results show that an increase in the electrical conductivity, as a function of tin content, reflects increasing density of the infinite cluster as demonstrated by the exponential dependence of electrical conductivity on the tin concentration.

Representative cluster structure of Sn component in overcritical region II is shown in Fig. 3. Tin creates

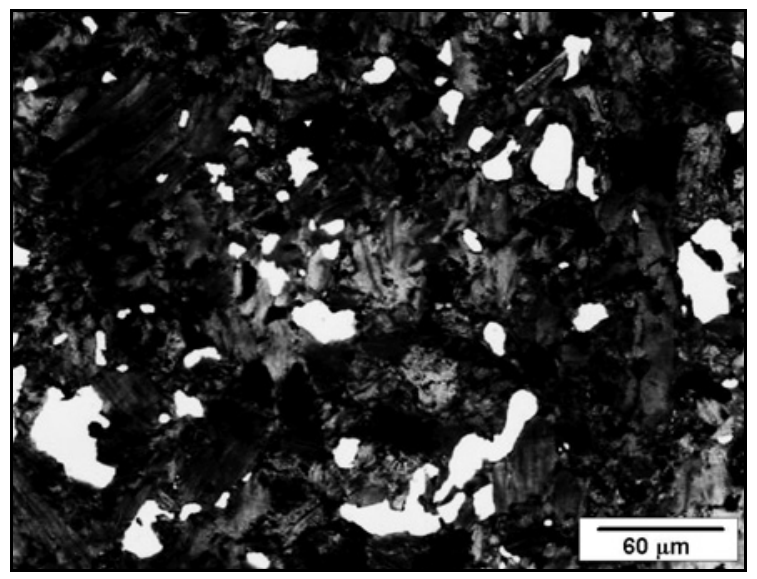

Fig. 2. Structure of Sn cluster in subcritical region I. Composite material containing $5.0 \mathrm{vol} . \% \mathrm{Sn}$.

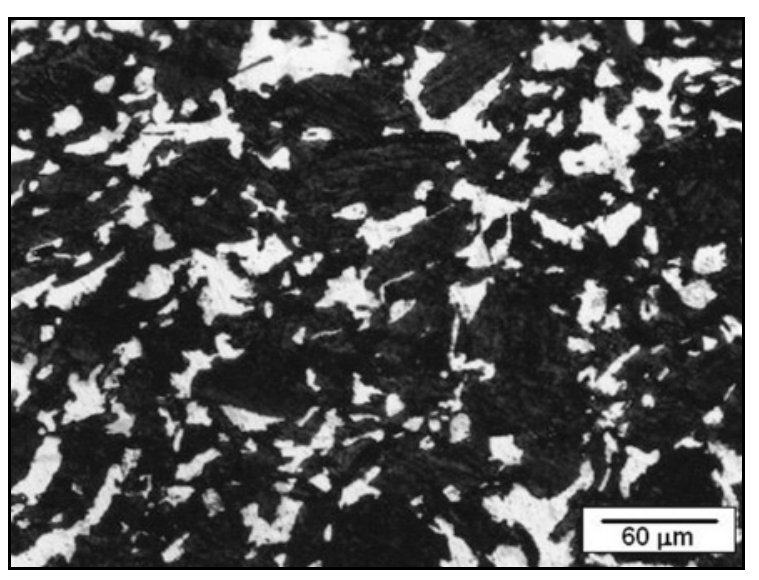

Fig. 3. Structure of Sn cluster in overcritical region II. Composite material containing 23.3 vol.\% Sn.

the conductive bonds responsible for the power dependence of electrical conductivity. The depicted sample contains 23.3 vol.\% Sn (bright areas) forming an infinite cluster.

Closer view on the composite microstructure can give the mercury porosimetry. The result of the porosimetry analysis of non-infiltrated carbon material is presented in Fig. 4. On the cumulative curve of $\mathrm{Hg}$ intrusion, one can see a threshold positioned at $3 \mu \mathrm{m}$. If the porosimetry result is compared with dimensions of pores filled with tin (Figs. 2 and 3 ) one can see that the result is not the same. It is because most of the pores have the "ink bottle" shape. The entrances into pores are the necks having smaller dimension than their diameter. These bottlenecks are important for connectivity of metallic clusters and critically influence the electrical conductivity.

On the other hand, the pores volume filled with metal is important for the formation of sufficiently 


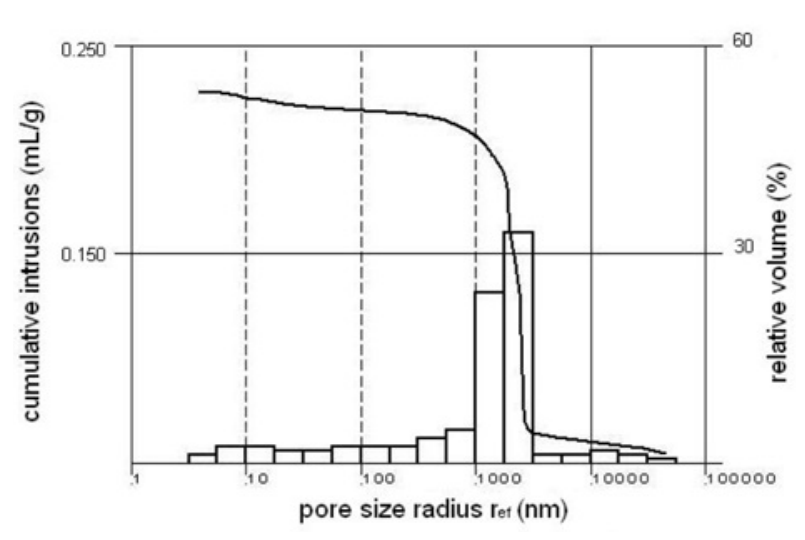

Fig. 4. The pore size distribution of the non-infiltrated carbon material.

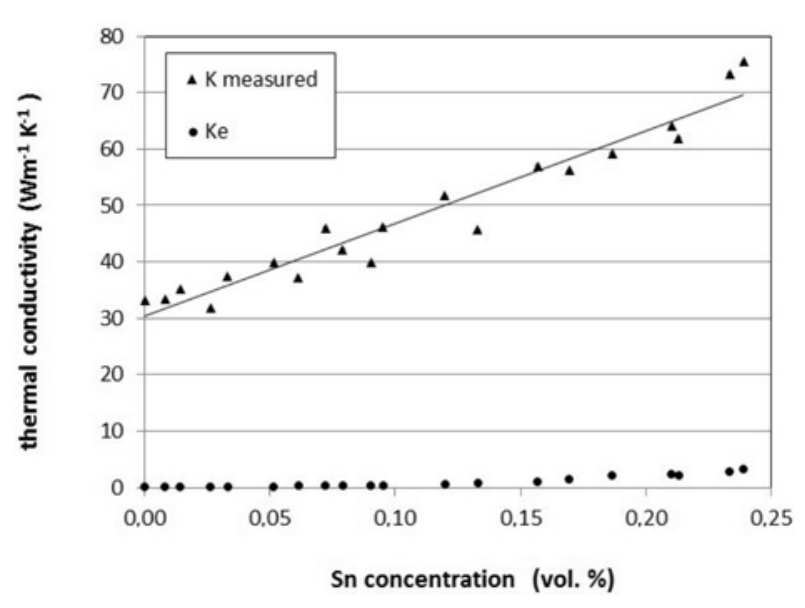

Fig. 5. Diagram of the thermal conductivity $K$ vs. Sn content (upper diagram) and calculated dependence of electron thermal conductivity $K_{\mathrm{e}}$ on Sn content (lower diagram).

large tin-carbon contact areas. On the porosimetry curve, two local maxima can be seen. The principal one at $3 \mu \mathrm{m}$ belongs to the pores formed between individual grains of carbon. The second one represents $10 \mathrm{~nm}$ pores residing inside individual particles of raw carbon material. Both results well correspond with the data reported in the literature [18].

The same composite samples were used for measurements of thermal conductivity as a function of tin content (Fig. 5). The diagram shows the gradual increase in thermal conductivity with increasing tin content. The dependence is linear at the low tin concentrations and at the highest concentrations it becomes slightly non-linear. Unlike the electrical conductivity, thermal conductivity dependence does not exhibit any significant change in the tin concentration above 7.9 vol. $\% \mathrm{Sn}$.

The difference between electrical and thermal conductivity dependence can be attributed to the low con- trast between thermal conductivities of the tin cluster and the carbon matrix. As a result, the heat flows through the metal-carbon interface without preferring any of the composite components so that the process is similar to electrical conductivity at concentrations $x \leq x_{\mathrm{c}}$. For the tin contents region of $x \leq x_{\mathrm{c}}$, the continuous electrical conductive cluster is not created, and the electron flow passes from one component to the other. As mentioned above (Sec. 1), the heat flow in the graphite material is primarily of a phonon type and the contribution of electrons can be neglected. As for the tin, the heat flow consists of the transport of electrons as well as phonons. The transport of electrons depends on the electrical potential gradient. The temperature gradient does not affect the electrical conductivity, but it may increase the thermal conductivity through movement of electrons.

There was calculated the thermal conductivity term $K_{\mathrm{e}}$ connected with the thermal movement of electrons by using the Wiedemann-Franz law wherein the values of specific electrical conductivity $\sigma_{\text {ef }}$ were used. A plot of electron thermal conductivity $K_{\mathrm{e}}$ vs. Sn concentration is shown in Fig. 5 (lower part of the diagram) indicating that thermal conductivity of electrons $K_{\mathrm{e}}$ is much lower than overall thermal conductivity $K$. This means that overall thermal flow is mostly at the expense of phonons contribution. This is in agreement with assumption presented in both the section 1 and the literature $[11,12]$. A bit higher contribution of electrons on thermal conductivity is seen at high tin concentrations ( $\sim 25$ vol.\% Sn). This is due to sharp increase in electrical conductivity for Sn content higher than $x_{\mathrm{c}}$ where the trend curve is changed from the linear to the power type (region II). As the heat flow is transferred from one component to another, it is important to characterize the role of interfaces. The interfaces are formed during composite fabrication when the liquid metal is pressed into porous carbon. Filling of pores can be expressed by the Washburn formula [4]:

$$
r_{\mathrm{ef}}=-\frac{2 \Gamma \cos \theta}{P_{\mathrm{c}}}
$$

where $r_{\text {ef }}$ is the effective pore radius, $\Gamma$ is the surface tension, $\theta$ is the carbon-tin wetting angle and $P_{\mathrm{c}}$ is the capillary pressure.

Equation (6) suggests that the smaller is the effective pore radius $r_{\text {ef, }}$, the greater capillary pressure $P_{\mathrm{c}}$ is needed. For the wetting angle $\theta=156^{\circ}$, which is experimentally measured for liquid tin wetting on a graphite surface [10], the capillary pressure $P_{\mathrm{c}}$ is negative and repulses liquid tin from pores so that spontaneous infiltration cannot occur. Thus, external gas pressure $P_{\text {ext }}=-P_{\mathrm{c}}$ has to be applied to intrude the metal into pores. At the same time increase in infiltration pressure leads to the enlargement of contact areas between components. During infiltration, 


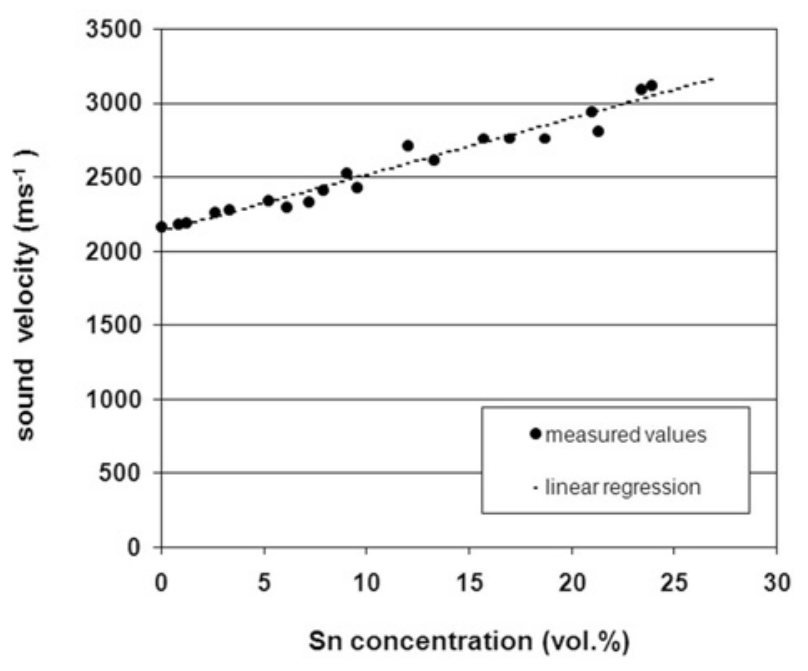

Fig. 6. Dependence of sound velocity on Sn volume fraction.

the pores with high $r_{\text {ef }}$ are filled first followed by the filling of smaller and smaller pores. Very small pores remain unfilled. Hence, the more metal penetrates into pores, the larger contact area is formed (Figs. 3 and 4).

Poor wetting at carbon-tin interfaces leads to the poor adhesion so that non-perfect interfacial bond is formed. Due to the poor wetting $(\cos \theta<0)$ the work of adhesion $W$ decreases according to the formula:

$$
W=\Gamma(1+\cos \theta)
$$

Drop in $W$ results in a non-perfect interfacial bond which influences the heat transport negatively through interfaces. In that regard negative role also plays volume shrinkage of the metal during solidification. When taking the volume shrinkage of $2.7 \%$ for Sn at the solidification [10], simple modelling suggests that in $20 \times 20 \times 20 \mu \mathrm{m}^{3}$ dimensioned pore element a gap of $0.23 \mu \mathrm{m}$ can be formed between the tin and the carbon generating very large local barrier for the heat transfer.

The dependence of the concentration of tin on the sound velocity of the composite is shown in Fig. 6 . The figure depicts a gradual rising of sound velocity as a function of an increasing tin content.

The dependence of longitudinal oscillations of velocity on tin concentrations is linear. In contrast to electrical and thermal conductivities sound velocity is not affected by electrons but by elastic oscillations of the composite. Both the sound velocity of tin and that of the carbon matrix measured at a zero concentration of tin are not in contrast because the values are very close. Therefore, at the critical tin concentration $x_{\mathrm{c}}$, no significant change in the linear dependence of the sound velocity occurred resembling the diagram of thermal conductivity. Irrespective of infinite cluster creation, longitudinal oscillations propagate in the composite body through interfaces under participation of both composite components. Like the case of thermal conductivity, an increase in contact areas between components influences the transfer of longitudinal oscillation positively. Moreover, the filling of pores with tin reduces the residual porosity of carbon body (Figs. 2 and 3) strongly thus facilitating the propagation of longitudinal oscillations. This is demonstrated in Fig. 6 where sound velocity increases with increase in tin content.

\section{Conclusions}

Interpenetrated tin-carbon composite materials with varying tin/carbon concentration ratios (up to 25 vol. $\% \mathrm{Sn}$ ) were prepared by the pressure infiltration technology. The composite samples were tested regarding specific electrical conductivity, thermal conductivity, and sound velocity as a function of tin content. At the tin concentration of 7.9 vol. $\%$ the linear dependence of specific electrical conductivity is changed to the power dependence due the electron percolation connected with the formation of the continuous tin cluster. As for the thermal conductivity, it is not influenced by the tin cluster structure exhibiting a linear dependence on the tin content in the whole concentration range. Similar linear dependence on the tin content was also observed for the sound velocity.

\section{References}

[1] Teng, H. S., Chang, Y. J., Hsieh, C. T.: CARBON, 39, 2001, p. 1981. doi:10.1016/S0008-6223(01)00027-6

[2] Li, X., Xue, J., Zhu, J., Zhang, Q.: In: Light Metals. Analysis of Porous Structures of Graphitic Cathode Materials and the Correlation to Penetrated Sodium. Ed.: Suarez, C. E. Hoboken, John Wiley \& Sons, Inc. 2012. doi:10.1002/9781118359259.ch228

[3] Sing, K. S. W., Everett, D. H., Haul, R. A. W., Moscou, L., Pierotti, R. A., Rouquerol, J., Siemieniewska, T.: Pure \& Appl. Chem., 57, 1985, p. 603. doi:10.1351/pac198557040603

[4] Kúdela, S., Schweighofer, A., Kunkela, J.: Composites Manufacturing, 3, 1992, p. 43. doi:10.1016/0956-7143(92)90182-T

[5] Kúdela, S., Schweighofer, A., Krcho, S.: In: Proc. of 6th Int. Symposium on Metallic Composite Materials. Ed.: Šebo, P. Bratislava, CSVTS-UMMS SAS 1986, p. 125.

[6] Najdič, Yu. V., Kolesničenko, G. A.: Investigation of Graphite and Diamond Wetting Liquid Metals. Kiev, Naukova Dumka 1967. (in Russian)

[7] Loebner, E. E.: Phys. Rev., 102, 1956, p. 46. doi:10.1103/PhysRev.102.46

[8] Sulepov, S. V.: Physics of Carbon Materials. Moscow, Izdatelstvo Metallurgija 1972. (in Russian) 
[9] Kelly, B. T.: Physics of Graphite. London-New Jersey, Applied Science Publishers 1981. doi:10.1016/0022-3115(83)90088-0

[10] Smithells, C. J.: Metals Reference Book. 7th Edition. London, Butterworths 1992.

[11] Taylor, R., Gilchrist, K. E., Poston, L. J.: CARBON, 6, 1968, p. 537. doi:10.1016/0008-6223(68)90093-6

[12] Krcho, S.: Study of Transport Phenomena of Carbon Skeleton Composites Infiltrated with Metals. [PhD. Thesis]. Bratislava, IMMM SAS 1987.

[13] Lide, R. L.: CRC Handbook of Chemistry and Physics. Int. Ver. 2005. Boca Raton, CRC Press 2005.

[14] Bhlapibul, S., Pruksathorn, K.: Korean J. Chem. Eng., 25, 2008, p. 1226. doi:10.1007/s11814-008-0203-2
[15] Chen, Y. H., Wang, C. W., Liu, G., Song, X. Y., Battagli, V. S., Sastry, A. M.: Journal of the Electrochemical Soc., 154, 2007, p. A978. doi:10.1149/1.2767839

[16] Emmer, S., Kováčik, J.: Scientific Proceedings Faculty of Mech. Eng., 19, 2011, p. 126. doi:10.2478/v10228-011-0021-0

[17] Broadbent, S. R., Hamersley, J. M.: Proceedings of the Cambridge Phil. Soc., 53, 1957, p. 629. doi:10.1017/S0305004100032680

[18] Fialkov, A. S.: Carbon and Graphite Materials. Moscow, ENERGIA 1979. (in Russian) 